Abstract-Long-term sustainable management of wild populations should be based on management actions that account for the genetic structure among populations. Knowledge of genetic structure and of the degree of demographic exchange between discreet populations allows managers to better define management units. However, adequate gene loci for population assessments are not always available. In this study, variable co-dominant DNA loci in the heavily exploited marine genus Brevoortia were developed with a microsatellite-enriched DNA library for the Gulf Menhaden (Brevoortia patronus). Microsatellite marker discovery was followed by genetic characterization of 4 endemic North American Brevoortia species, by using 14 novel loci as well as 5 previously described loci. Power analysis of these loci for use in species identification and genetic stock structure was used to assess their potential to improve the stock definition in the menhaden fishery of the Gulf of Mexico. These loci could be used to reliably identify menhaden species in the Gulf of Mexico with an estimated error rate of $\alpha=0.0001$. Similarly, a power analysis completed on the basis of observed allele frequencies in Gulf Menhaden indicated that these markers can be used to detect very small levels of genetic divergence $\left(F_{\text {st }} \approx 0.004\right)$ among simulated populations, with sample sizes as small as $n=50$ individuals. A cursory analysis of genetic structure among Gulf Menhaden sampled throughout the Gulf of Mexico indicated limited genetic structure among sampling locations, although the available sampling did not reach the target number $(n=50)$ necessary to detect minimal values of significant structure.

Manuscript submitted 14 February 2013. Manuscript accepted 6 December 2013. Fish. Bull. 112:71-81 (2014).

doi: $10.7755 /$ FB.112.1.5

The views and opinions expressed or implied in this article are those of the author (or authors) and do not necesarily reflect the position of the National Marine Fisheries Service, NOAA.

\title{
Limited genetic structure of Gulf Menhaden (Brevoortia patronus), as revealed by microsatellite markers developed for the genus Brevoortia (Clupeidae)
}

\author{
Joel D. Anderson (contact author) \\ William J. Karel \\ Email Address for contact author: joel.anderson@tpwd.state.tx.us \\ Perry R. Bass Marine Fisheries Research Station \\ Texas Parks and Wildlife Department \\ 3862 FM 3280 \\ Palacios, Texas 77465
}

Management of wild populations is improved when management actions take into account genetic structure. Stock assessments of marine species are improved when genetic data are used in the development of a fishery stock definition (Begg and Waldman, 1999; Waples et al., 2008). Different outcomes in stock assessments are expected between a scenario with a single exploited population and a scenario in which 2 or more demographically independent populations are exploited simultaneously by a fishery. In the latter case, treating 2 or more independent fishery stocks as a single unit could potentially lead to underestimates of the effects of exploitation on local stocks and an overall diminished yield (Carvalho and Hauser, 1994). Therefore, genetic analyses of population structure can improve stock assessments by increasing accuracy in identification of the number of populations exploited and the extent and direction of demographic exchange (migration) among exploited populations (Begg and Waldman, 1999; Waples et al., 2008).

A recent Southeast Data, Assessment, and Review (SEDAR) stock assessment indicated that more genetic data are needed to appropriately define the stock harvested by the menhaden fishery (Brevoortia spp.) in the Gulf of Mexico (Vaughn et al. ${ }^{1}$ ). Two

\footnotetext{
1 Vaughn, D., A. Schueller, J. Smith, and S. VanderKooy (eds.). 2011. SEDAR 27: Stock assessment report, Gulf of Mexico
}

aspects of the stock definition for the menhaden fishery can be improved with genetic data. First, the menhaden catch in the Gulf of Mexico is thought to consist of 3 species: Gulf Menhaden (Brevoortia patronus), Finescale Menhaden (B. gunteri), and Yellowfin Menhaden (B. smithi). Ahrenholz (1981) suggested that 99\% of the catch comprised Gulf Menhaden, but this finding can be re-assessed with sampling of diagnostic microsatellite DNA loci. Second, information about population structure within the species targeted by the fishery, Gulf Menhaden, will result in a better definition of management units that compose the stock (Waples et al., 2008). A more suitable stock definition in turn will result in more practical biological reference points in stock assessments. Previous studies of population structure in Gulf Menhaden have indicated that there is a single stock in the Gulf of Mexico, but these studies were completed with sampling designs that were not specifically intended to assess fine-scale population structure among locales in the Gulf of Mexico (Bowen and Avise, 1990; Anderson, 2007; Anderson and Karel, 2007). A major downfall of these studies has been a paucity of adequate gene loci developed for the genus Brevoortia,

menhaden, 460 p. Southeast Data, Assessment, and Review, Charleston, SC. [Available from http://www.sefsc.noaa. gov/sedar/Index.jsp.] 
and this has ultimately resulted in limited resolution in addressing population structure and hybridization among Brevoortia congeners in the Gulf of Mexico (Anderson, 2007; Anderson and Karel, 2007; Anderson and McDonald, 2007).

The first step in addressing population structure among wild populations is the identification of appropriate polymorphic loci. Adequate loci with sufficient polymorphism are required to definitively detect patterns of divergence from limited sample sizes. When low levels of genetic divergence have occurred between populations, as might be expected in Gulf Menhaden, the total number of gene loci employed, as well as the average number of alleles across loci, is predictive of the power in detection of that divergence (Kalinowski, 2004; Bernatchez and Duchesne, 2000).

Additionally, gene loci should have a limited amount of extraneous "noise" that can be created by assayspecific genotype errors (for instance, size homoplasy, null alleles, or allele dropout). Many published primer sets intended for use with wild populations generally consist of an initial data analysis from a single, small sample ( $n \leq 20$ individuals) and, therefore, may either lack polymorphism or include one or more genotyping inconsistencies that are not identified in the initial marker characterization. Furthermore, primer sets for nonmodel organisms can be difficult to find in the published literature, and the lack of such primer sets commonly results in use of heterologous gene loci from model organisms or ecologically important congeners (Rico et al., 1996). Heterologous loci have been used commonly in nonmodeled organisms, but the genetic variability of these loci in nontarget species is usually lower than the genetic variability in target species (Ellegren et al., 1997) and the evolutionary properties of the repeat motif itself (such as motif sequence or pattern) are unknown.

This study attempts to address the lack of adequate genetic loci for the genus Brevoortia generally and for Gulf Menhaden in particular. A genomic library of Gulf Menhaden restriction fragments was enriched for novel short tandem repeats (STRs, or microsatellites), and microsatellite markers were identified by using a published method (Glenn and Schable, 2005). The discriminatory power of the resulting group of microsatellite loci in the Gulf Menhaden population assignment was quantitatively assessed, as was the likelihood of species identification through the use of a subset of diagnostic loci. These loci increase the genetic tools available for improvement of stock definitions in the Gulf Menhaden and other species of Brevoortia.

\section{Materials and methods}

\section{Study area and sampling methods}

Specimens of Brevoortia spp. were collected throughout the North American range of this genus and were identified using the morphological characteristics described in Dahlberg (1970). For the initial phase of the study (microsatellite identification), genomic DNA was isolated from 2 Gulf menhaden juveniles $(<40 \mathrm{~mm}$ total length) collected inshore in the vicinity of Freeport, Texas (Fig. 1) in spring 2012. These individuals were collected with a bag seine along the shoreline. The second phase of the study was carried out with previously sampled individuals from Anderson (2007). These fish included 118 Gulf Menhaden individuals from 4 sampling locales in the Gulf of Mexico sampled between 2002 and 2003: Laguna Madre, Texas, 2003 [ $n=29$ ]; Matagorda Bay, Texas, 2003 [ $n=30]$; Lake Charles, Louisiana, 2002 [ $n=29]$; and Apalachicola, Florida, 2003 $[n=30])$. Samples also included 30 Atlantic Menhaden (Brevoortia tyrannus) from Sandy Hook Bay, New Jersey, collected in 2002; 8 Atlantic Mehaden from Bogue Sound, North Carolina, collected in 2002; 22 Finescale Menhaden from Sabine Pass, Texas, collected in 2004; and 19 Yellowfin Menhaden from Charlotte Harbor, Florida, collected in 2003. Because these samples were collected across multiple years, it was expected that multiple independent cohorts were collected.

\section{Library enrichment and microsatellite loci cloning}

A microsatellite-enriched library of Gulf Menhaden genomic DNA was constructed and microsatellite loci were cloned by using the protocol of Glenn and Schable (2005). Genomic DNA was isolated from 2 juvenile Gulf Menhaden sampled in Freeport, Texas, with a Gentra Puregene Tissue Kit (Qiagen, Inc., Valencia, $\mathrm{CA}^{2}$ ), and DNA was eluted in $50 \mu \mathrm{L}$ of TE buffer (10 mM Tris, 1 mM EDTA). Each DNA sample was then cut with $R s a \mathrm{I}$ restriction enzyme, resulting in fragments in a range of 200-1000 base pairs (bp). Restriction fragments from each sample were then combined into a single pool and ligated to biotin-labeled SuperSNX linkers (Glenn and Schable, 2005). Linked fragments were enriched for microsatellite repeat-bearing fragments with biotin-capture Dynabeads (Life Technologies Corp., Carlsbad, CA) in 2 enrichment mixtures: a trinucleotide mix (AAC, AAG, AAT, ACT, ATC) and a tetranucleotide mix number (AAAC, AAAG, AATC, AATG, ACAG, ACCT, ACTC, ACTG). Successive temperature steps of $\mathrm{T}_{\mathrm{m} 1}=48.5^{\circ} \mathrm{C}$, and $\mathrm{T}_{\mathrm{m} 2}=58.5^{\circ} \mathrm{C}$ were used for enrichment. Enriched fragments were ligated into plasmids and transformed by using Invitrogen TOPO-TA cloning kits (Life Technologies Corp.), according to the manufacturer's instructions. Transformed bacteria were plated onto 150$\mathrm{mm}$ ampicillin-inoculated S-Gal agar plates (Sigma-Aldrich Co., St. Louis, MO) and grown for 2 days at $37^{\circ} \mathrm{C}$. Positive colonies were selected and stored in $50 \mu \mathrm{L}$ of TE buffer before DNA sequencing.

\footnotetext{
2 Mention of trade names or commercial companies is for identification purposes only and does not imply endorsement by the National Marine Fisheries Service, NOAA
} 


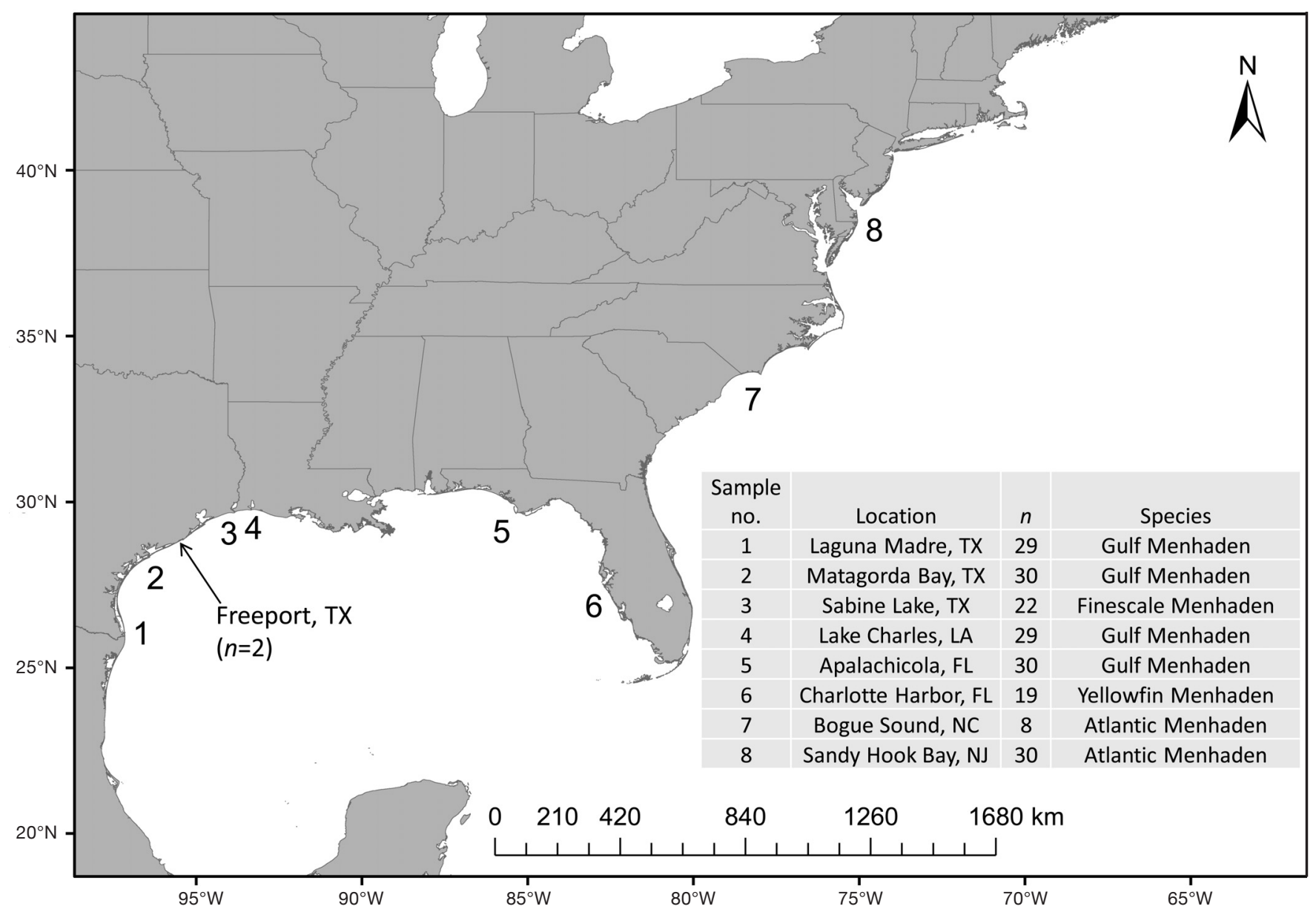

Figure 1

Map of sampling locations in the Gulf of Mexico and Atlantic Ocean where the samples of Gulf Menhaden (Brevoortia patronus), Finescale Menhaden (B. gunteri), Yellowfin Menhaden (B. smithi), and Atlantic Menhaden (B. tyrannus) were collected for this study. Samples used for microsatellite identification were collected with a seine net along the shoreline near Freeport, Texas, in 2012. All other samples used for power analysis and analysis of population genetics were taken as described in a previous study (Anderson, 2007) in 2002-04.

Positive colonies were amplified through polymerase chain reaction (PCR) with M13 primers (Life Technologies Corp.) before sequencing. Each reaction included 1 $\mu \mathrm{L}$ of template DNA (50 ng/ $\mu \mathrm{L}), 1$ Ready-To-Go ${ }^{\circledR}$ PCR bead (GE Healthcare Life Sciences, Piscataway, NJ), and $24 \mathrm{\mu L}$ of forward and reverse primer cocktail (0.4$\mu \mathrm{M}$ standard primer concentration of each primer), for a total of $25 \mu \mathrm{L}$. The following cycling regime was used for PCR: 1) 1 cycle of $2 \mathrm{~min}$ at $95^{\circ} \mathrm{C}$; 2) 10 cycles of $95^{\circ} \mathrm{C}$ for $30 \mathrm{~s}, 55^{\circ} \mathrm{C}$ for $30 \mathrm{~s}$ decreasing $1^{\circ} \mathrm{C}$ each cycle, and $72^{\circ} \mathrm{C}$ for $1 \mathrm{~min}$; 3) 20 cycles of $95^{\circ} \mathrm{C}$ for $30 \mathrm{~s}, 50^{\circ} \mathrm{C}$ for $30 \mathrm{~s}$, and $72^{\circ} \mathrm{C}$ for $1 \mathrm{~min}$, adding $3 \mathrm{~s}$ of extension per cycle; and 4) a final extension period of $7 \mathrm{~min}$ at $72^{\circ} \mathrm{C}$. Aliquots of each PCR product were purified with Exo-Sapit ${ }^{\circledR}$ PCR purification reagent (Affymetrix, Inc., Santa Clara, CA). Purified amplicons were sequenced with M13 primers with the following reaction conditions: 30 cycles of $96^{\circ} \mathrm{C}$ for $20 \mathrm{~s}, 50^{\circ} \mathrm{C}$ for $20 \mathrm{~s}$, and $60^{\circ} \mathrm{C}$ for $4 \mathrm{~min}$. Sequencing reactions were carried out in 10$\mu \mathrm{L}$ volumes with DTCS Quick Start Master Mix (Beckman Coulter Inc., Brea, CA) according to the manufacturer's instructions. Sequences were separated with a CEQ 8000 capillary sequencer (Beckman Coulter, Inc.). Raw sequences were trimmed and edited manually and overlapping forward and reverse traces for each sequence were aligned with the software package Sequencher (vers. 4.2; Gene Codes Corp., Ann Arbor, MI).

\section{Primer design and locus characterization}

Among 573 positive clones examined, 91 contained repeated fragments, meaning the microsatellite motif was repeated $\geq 5$ times. From the 91 prospective loci, primers were designed for the loci that had the following characteristics: 1) the repeated motif was 3 bases or more in length, 2) no interruptions occurred within 
the repeated segment, and 3) at least 20 unambiguously sequenced base pairs were on both sides of the repeated motif, enabling primer design. Primers were constructed manually for the 40 resulting loci, and these primers were used to genotype a panel of 16 Gulf Menhaden individuals (from Matagorda Bay, TX) and 8 Atlantic Menhaden individuals (from Bogue Sound, NC) by using the initial PCR conditions described above. Each primer set was labeled with a WellRed fluorescent dye (primer manufacture and labeling by Sigma-Aldrich Co.), and used to amplify approximately $100 \mathrm{ng}$ of genomic DNA through the use of PCR. Amplified products were combined with a 400-bp size standard and separated with a CEQ 8000 sequencer (Beckman Coulter, Inc.), with default (Frag-1) analysis parameters. Fragment analysis was performed with Beckman Coulter software, and all allelic bins (estimated allele size in base pairs) were established using Beckman Coulter fragment analysis software, with default values for fragment analysis.

The following qualitative criteria were used to choose a final group of microsatellites from the 40 original loci: 1) primer pairs amplified a product in a majority of Gulf Menhaden individuals, 2) alleles were spaced as expected on the basis of the original clone sequences (i.e., trinucleotide motifs were 3 bases apart, tetranucleotide motifs were 4 bases apart), 3) peak intensity appeared to be similar between heterozygote peaks (i.e., there was no visual evidence for allele dropout), 4) alleles were scored with minimum stutter, and 5) preference was given to loci that produced PCR amplicons in both species. This elimination process resulted in 14 loci retained from the 40 original loci examined. An additional 5 loci initially developed for American Shad (Alosa sapidissima) (Waters et al., 2000) and previously assayed in Brevoortia spp. (Anderson, 2007) were included in the final group to increase the sampling size of the group and to compare the performance of the new loci to a previously published set. All sampled individuals were genotyped by using these 19 loci.

\section{Micro-Checker and genetic polymorphism}

The software program Micro-Checker (Van Oosterhout et al., 2004) was used to test each locus for evidence of null alleles and genotyping errors. Each marker was tested independently in all 4 endemic North American species of Brevoortia: Gulf Menhaden, Atlantic Menhaden, Yellowfin Menhaden, and Finescale Menhaden. Data from each of the 4 Gulf Menhaden sampling locales also were treated as independent samples and analyzed separately. If evidence was found for a null allele in any sample and locus combination, the frequency of the null allele was calculated as the average of the estimates from 3 independent methods (Chakraborty et al., 1992; Brookfield, 1996; Van Oosterhout et al., 2006). Micro-Checker also was used to assess the statistical likelihood of genotyping errors caused by either allele stutter (one or more PCR artifacts being genotyped as true peaks) or allele dropout (alleles that amplify poorly and, therefore, are not properly genotyped).

The likelihood ratio test of Excoffier and Slatkin (1998) was used to test for linkage disequilibrium between all pairs of microsatellite loci in Gulf Menhaden. Testing was done through the use of the software program Arlequin (vers. 3.5; Excoffier et al., 2005) and statistical significance assessed with 1000 data permutations. The level of statistical significance was adjusted for each comparison of linkage disequilibrium by using the sequential Bonferroni procedure (Rice, 1989). Each marker also was tested for deviation from Hardy-Weinberg equilibrium (HWE), with the expectation that loci with null alleles would likely also deviate from HWE. The exact test method of Guo and Thompson (1992) was used for HWE tests with a Markov chain length of 100,000 steps, as implemented in Arlequin. After the exact test, statistical significance was assessed at each locus with the $P$-value of the test statistic, and statistical significance is reported here both before and after sequential Bonferroni adjustment (Rice, 1989).

The observed and expected heterozygosity and the number of alleles also were estimated for each locus, in each species, with Arlequin. Differences in genetic variability among species were tested by comparing expected heterozygosity $\left(H_{\mathrm{e}}\right)$ among species. Each locus was treated as an independent data point, and the null hypothesis that $H_{\mathrm{e}}$ in Gulf Menhaden was not statistically different from $H_{\mathrm{e}}$ in other species of Brevoortia was tested with a Wilcoxon nonparametric signed-rank test over all loci. The Wilcoxon test was performed in $\mathrm{R}$ software (vers. 2.14.1; R Development Core Team, 2011), with the wilcox.test function. To further compare levels of polymorphism across loci and species, the polymorphic information content (PIC; Botstein et al., 1980) of each marker was estimated from observed allele frequencies in each species. The PIC statistic is a function of both allele number and frequency and, therefore, is a better estimator of discriminatory power than is the number of alleles alone.

The PIC was calculated in $\mathrm{R}$ for each locus with the formula

$$
P I C_{\mathrm{j}}=1-\sum_{i=1}^{n} p_{\mathrm{i}}^{2},
$$

where $p=$ the frequency of allele $i$ at locus $j$.

The Wilcoxon nonparametric signed-rank test was used to determine whether Gulf Menhaden had significantly higher PIC scores across all loci than the other Brevoortia species.

The relative power of assignment of Brevoortia gene loci was tested with the program Whichloci (Banks et al., 2003). The Whichloci method is a resampling technique that generates simulated populations from observed allele frequencies in experimental samples, and then assigns experimental individuals to populations on the basis of the likelihood of an individual's genotype in each population. The accuracy of assignment 
can be assessed at any level of assignment stringency, and that level is set by the user as a LOD (log odds) score. Individuals from different samples within species were combined, and the relative power of the loci in the assignment of individuals to species was assessed. Loci that deviated from HWE or that failed to amplify any species were excluded from species assignment. As a result, assignment was performed with 14 loci (see the Results section).

During species assignment, marker performance was assessed at various levels of $\mathrm{LOD}=2,3$, and 4 ; these levels of stringency are approximately analogous to an acceptable error rate of $0.01,0.001$, and 0.0001 , respectively. Simulated population sizes were set at $n=100$, and 100 replicate simulations were performed. Two properties of the genetic data set were assessed after simulation and assignment: 1) the number of loci needed to correctly assign $100 \%$ of individuals to species at the predetermined level of stringency and 2) the relative contribution (locus score; Banks et al., 2003) of each locus in the assignment of individuals when LOD was set at 1 . It is anticipated that within the menhaden fishery of the Gulf of Mexico, the Atlantic Menhaden is rarely or never encountered. Therefore, the Atlantic Menhaden was excluded from the analysis.

The power of these loci to reveal small levels of population differentiation within Gulf Menhaden was explored through a Powsim analysis (Ryman and Palm, 2006). Allele frequencies were estimated from composite data in the Gulf Menhaden samples with Fstat, vers. 2.3, (Goudet, 1995) and were used to perform 500 replicated experiments on 2 simulated populations. Because of the uncertainty involved in accurately estimating and accounting for null alleles, loci that deviated significantly from HWE in Gulf Menhaden and had estimated null allele frequency $p \geq 0.10$ were excluded. As a result, power analysis was performed with 15 loci (see the Results section).

The 2-population model was chosen because it is the simplest mode of population structure, and previous studies of marine finfish in the Gulf of Mexico have frequently indicated a marine "suture zone" east of the Mississippi River (Portnoy and Gold, 2012), resulting in eastern and western Gulf of Mexico stocks of several species or species pairs. Two levels of sampling $(n=40$ and 50 individuals) were conducted after 10 generations of genetic drift, with the assumption of effective population sizes of 500, 1000, and 5000 individuals (these values equate to $F_{\text {st }}=0.01,0.004$, and 0.001 , respectively). The power of the data at each level of $N_{\mathrm{e}}$ and at each sample size was assessed as the number of significant results obtained across all 500 simulations, assessed with the Fisher's exact test.

\section{Genetic structure of Gulf Menhaden}

Among the 4 Gulf Menhaden samples, evidence for nonrandom associations of alleles or genotypes (genetic structure) was assessed with the program Structure (vers. 2.3.4; Pritchard et al., 2000). Models that included $K=1-4$ potential populations were evaluated through comparison of the posterior probability of each model (where $K$ is the prior value for the assumed number of populations represented by the data). Each level of $K$ was assessed with 50,000 burn-in iterations, followed by 450,000 sampling iterations. Prior evaluations of Gulf Menhaden have indicated a high level of gene flow among samples from the Gulf of Mexico; therefore, we used the default Structure model, assuming historical admixture at each level of $K$, while also assuming allele frequencies among locales were correlated. Admixture coefficient stability among individuals during the course of runs in Structure was used to evaluate model convergence. Loci that deviated from HWE or that had null alleles of estimated frequency $p \geq 0.10$ were excluded from all genetic structure analyses.

Genetic structure was further explored by using the analysis of molecular variance (AMOVA; Excoffier et al., 1992). The initial AMOVA model was used to test for significant genetic divergence among Gulf Menhaden samples $\left(F_{\text {st }}\right)$, and secondarily among individuals within samples $\left(F_{\text {is }}\right)$. The significance of variance components in the AMOVA model were evaluated by 1000 permutations of the data, and $F$-statistics were estimated directly from variance components.

Finally, a Mantel test was used to assess the evidence of isolation-by-distance (IBD) among samples. Geographic association between sampling areas were calculated among sites by using shortest linear shoreline distance. This distance was correlated with Slatkin's linearized $F_{\text {st }}$ (Slatkin, 1995) by using the Mantel test. The significance of the coefficient of matrix correspondence $(r)$ was assessed by 1000 permutations of the data. The Mantel test and permutation procedure were conducted with Arlequin.

\section{Results}

The original 40 loci that were examined by means of the microsatellite discovery phase have been deposited in the National Center for Biotechnology Information Genbank database (accession numbers KC331110KC331149). The 14 loci that were retained from the 40 original loci through the use of qualitative criteria are listed in Table 1, and they will be discussed hereafter, along with 5 previously described loci (Waters et al., 2000). Among this final group of microsatellites, there were 6 tetranucleotide repeats, 9 trinucleotide repeats (1 trinucleotide repeat was initially described in $\mathrm{Wa}-$ ters et al., 2000), and 4 dinucleotide repeats (all dinucleotide repeats were initially described in Waters et al., 2000). Two of the tetranucleotide repeats unexpectedly contained alleles that were 2 bases apart (BP017, BP039). These alleles were not sequenced directly, but, in both cases, there were very short dinucleotide repeats adjacent to the targeted tetranucleotide repeats in the original clone sequence. It is likely that, in both 


\section{Table 1}

Locus name, primer sequences, microsatellite motif, and expected product length (in base pairs) for the 14 novel microsatellite loci developed from a Gulf Menhaden (Brevoortia patronus) genomic DNA library. The library was constructed using two individuals collected with a bag seine along the shoreline near Freeport, Texas, in spring 2012. Each locus has a GenBank accession number that can be accessed at the National Center for Biotechnology Information (www.ncbi.nlm.nih.gov; accessed 16 December 2012).

\begin{tabular}{|c|c|c|c|c|c|}
\hline Locus & Forward primer & Reverse primer & Motif & $\begin{array}{c}\text { Expected } \\
\text { PCR } \\
\text { length }\end{array}$ & $\begin{array}{c}\text { GenBank } \\
\text { accession } \\
\text { number }\end{array}$ \\
\hline Bp003 & ACTGCATGGTCACTAGCACT & CACCACTGCACCGCAGCATGCTGTC & (CAGA) 9 & 170 & KC331110 \\
\hline Bp017 & CATTTGGATCAAGAGTATCTATCC & CATTCTGCCACACCTCACATAGG & (TCTG) 5 & 150 & KC331112 \\
\hline Bp039 & GCGTTGACGCGGAGTAATGATCCAG & GATCTCGACTGACTTACAATGCGGC & (GTGA)6 & 220 & KC331113 \\
\hline Bp121 & ACTCAAAAGGGCCTGTCTCACGGAG & GCTGATTATAGTCTATAGGTTGGAC & (TCTA) 19 & 200 & KC331117 \\
\hline Bp155 & GAACTAGCATGGCAGCTAGATCTAG & GAACCATCAGAACAACTCAACCTGC & (ATC)13 & 150 & KC331119 \\
\hline Bp221 & TATTGCTGAGCCTGCTTGGCAG & GCCAAGCTGCTGCAGTCCCAGCAGG & $(\mathrm{GTT}) 19$ & 210 & KC331123 \\
\hline Bp230 & CCAAGGACACATGCCCATATGTCTC & GTCTTCTGAATGGACCGAGTCTGGC & (TCA)16 & 150 & KC331124 \\
\hline Bp239 & CAATTTGTGAATCTCCAGGTCAG & GAACGCCAATCACAGCCTGGCAGTG & (CAA) 19 & 160 & KC331127 \\
\hline Bp275 & TTACGCGCCTGGTGTAGCCTAGCTC & CCTACGTGCGGCTCACATGTCTGG & (ATCAGA) 9 & 170 & KC331130 \\
\hline Bp301 & CATTATAGCTACAGAGCGGCTGG & CTGAGTGGGGTTCACCGCAGTCTG & $($ GATA $) 12$ & 150 & KC331134 \\
\hline Bp473 & CGGCACTCCGTGAACACAGCGG & ATGTGCTCTTTGGCTGCAG & $(\mathrm{CACT}) 8$ & 170 & KC331144 \\
\hline Bp489 & AGCGAGGTGAATGGTTCAATTCCAG & GCAAAACAATTGCCCATACTGGGAC & (TAG) 10 & 250 & KC331145 \\
\hline Bp501 & ACTCTCGCTGTGTTATTGACAGCGC & TCAGTTGACTGTGATCTCATGCGAC & (ATT/GTT) 15 & 150 & KC331146 \\
\hline Bp531 & CAGGTCTTTGTGACATGCCAAGCTG & CTGACTGCTTGAGGGATCGCAGCAG & (ATG/GTG) 16 & 140 & KC331148 \\
\hline
\end{tabular}

cases, the 2-base alleles reflect variation in the untargeted sequence adjacent to the targeted repeat motif. All alleles at these loci were nevertheless scored unequivocally through the use of Beckman Coulter fragment analysis software, and they were treated as dinucleotide repeats in downstream analyses.

In all 4 samples of Gulf Menhaden, 3 of 19 loci revealed evidence of null alleles (Table 2). These loci were BP017 (overall null allele frequency $p_{\mathrm{n}}=0.17$ ), BP301 (overall null $p_{\mathrm{n}}=0.25$ ), and AF039660 (overall null $p_{\mathrm{n}}=0.30$ ). The loci BP301 and AF039660 also showed evidence of genotyping errors due to excessive stutter in multiple populations. Four remaining loci revealed evidence of null alleles in at least 2 populations: BP155 (overall null $p_{\mathrm{n}}=0.05$ ), BP239 (overall null $p_{\mathrm{n}}=0.03$ ), BP275 (overall null $p_{\mathrm{n}}=0.06$ ), and AF039661 (overall null $p_{\mathrm{n}}=0.10$ ). A final 2 loci (AF039658 and AF049462) revealed evidence of null alleles at very low frequency $\left(p_{\mathrm{n}}<0.05\right)$ in single populations.

The locus BP155 failed to amplify in samples of Atlantic Menhaden, Finescale Menhaden, or Yellowfin Menhaden. There was evidence for null alleles in Atlantic Menhaden at the loci BP017, BP230, BP239, BP301, AF039658, AF0399660, and AF049462 (Table 3). In Finescale Menhaden, 2 loci showed evidence of null alleles (BP301, AF039660). In Yellowfin Menhaden, there was evidence of null alleles at only a single locus, BP301.

Within Gulf Menhaden, there was no evidence for linkage disequilibrium between any pair of loci. Across all loci, there was a range of 6 observed alleles (BP489,
AF039661) to 26 observed alleles (AF049462), with a mean of 14.8 alleles per locus (Table 2). When loci that had null alleles were included, there was an average observed heterozygosity of $H_{0}=0.64$, and an average expected heterozygosity of $H_{\mathrm{e}}=0.74$. With null loci removed, both observed and expected heterozygosity averaged $H=0.71$. Eight loci deviated significantly from Hardy-Weinberg expected genotypes before Bonferroni adjustment. Each of these disequilibrium loci indicated evidence of null alleles. After Bonferroni adjustment, only 4 loci significantly deviated from HWE. These 4 disequilibrium loci (BP017, BP301, and AF039660, AF039661) also had the highest estimated null allele frequency among all loci examined $\left(p_{\mathrm{n}} \geq 0.10\right.$ in each case).

Within other species of Brevoortia, overall genetic variability was greatly reduced, in comparison to Gulf Menhaden, for the genetic loci examined. The mean expected heterozygosity was $H_{\mathrm{e}}=0.63$ in Atlantic Menhaden, $H_{\mathrm{e}}=0.37$ in Yellowfin Menhaden, and $H_{\mathrm{e}}=0.44$ in Finescale Menhaden. Heterozygosity across all loci was significantly higher in Gulf Menhaden than in the 3 Brevoortia congeners on the basis of the Wilcoxon test $(P<0.001$, in each case). The mean PIC values for Atlantic Menhaden, Yellowfin Menhaden, and Finescale Menhaden were $0.617,0.356$, and 0.429 , respectively (compared with a PIC value of 0.734 for Gulf Menhaden). The PIC value across loci was significantly higher in Gulf Menhaden than in any of the other 3 Brevoortia species on the basis of the Wilcoxon test $(P<0.001$, in each case). 


\section{Table 2}

Statistics for 19 polymorphic microsatellite loci in Gulf Menhaden (Brevoortia patronus) collected throughout the Gulf of Mexico in 2002-04. The statistics include the overall number of alleles observed; heterozygosity, both observed $\left(H_{\mathrm{o}}\right)$ and expected $\left(H_{\mathrm{e}}\right)$; P-value of HardyWeinberg equilibrium (HWE) exact tests; null allele presence and frequency; and polymorphic information content (PIC). Statistical significance of the HWE $P$-value (initial $\alpha=0.05$ ) was assessed before (*) and after (**) sequential Bonferroni correction. Loci in italic type were described originally in a study of American Shad (Alosa sapidissima) (Waters et al., 2000).

\begin{tabular}{lccccccc}
\hline Locus & Alleles & $H_{\mathrm{o}}$ & $H_{\mathrm{e}}$ & HWE $(P)$ & Null alleles & Null freq. & PIC \\
\hline Bp003 & 15 & 0.627 & 0.690 & 0.227 & no & na & 0.687 \\
Bp017 & 10 & 0.364 & 0.666 & $0.000^{* *}$ & yes & 0.17 & 0.664 \\
Bp039 & 7 & 0.653 & 0.613 & 0.081 & no & na & 0.611 \\
Bp121 & 15 & 0.847 & 0.851 & 0.794 & no & na & 0.847 \\
Bp155 & 16 & 0.778 & 0.892 & $0.017^{*}$ & yes & 0.05 & 0.888 \\
Bp221 & 17 & 0.847 & 0.850 & 0.663 & no & na & 0.846 \\
Bp230 & 17 & 0.863 & 0.908 & 0.149 & no & na & 0.905 \\
Bp239 & 21 & 0.838 & 0.935 & $0.014^{*}$ & yes & 0.03 & 0.931 \\
Bp275 & 24 & 0.763 & 0.919 & $0.017^{*}$ & yes & 0.06 & 0.915 \\
Bp301 & 12 & 0.414 & 0.867 & $0.000^{* *}$ & yes & 0.25 & 0.863 \\
Bp473 & 7 & 0.615 & 0.624 & 0.077 & no & na & 0.622 \\
Bp489 & 6 & 0.085 & 0.099 & 0.213 & no & na & 0.098 \\
Bp501 & 15 & 0.907 & 0.883 & 0.713 & no & na & 0.880 \\
Bp531 & 19 & 0.915 & 0.907 & 0.711 & no & na & 0.903 \\
AF039657 & 15 & 0.686 & 0.690 & 0.916 & no & na & 0.687 \\
AF039658 & 21 & 0.763 & 0.868 & 0.101 & yes & 0.03 & 0.865 \\
AF039660 & 13 & 0.271 & 0.666 & $0.000^{* *}$ & yes & 0.30 & 0.663 \\
AF039661 & 6 & 0.102 & 0.138 & $0.001^{* *}$ & yes & 0.10 & 0.137 \\
AF049462 & 26 & 0.831 & 0.930 & $0.011^{*}$ & yes & 0.02 & 0.926 \\
Overall & 14.8 & 0.641 & 0.737 & - & - & - & - \\
& & & & & & &
\end{tabular}

Species assignment was carried out with 14 genetic loci, excluding the 4 loci that deviated from HWE in Gulf Menhaden after Bonferroni adjustment (BP017, BP301, AF039660, and AF039661) and also excluding the locus that did not amplify a product in species other than Gulf Menhaden (BP155). Ten of the 14 loci contributed more than $95 \%$ to the cumulative locus score in species assignment (Table 4). Interestingly, the microsatellite that ranked highest in species assignment (AF039657) was initially developed for use in American Shad (Waters et al., 2000). With a stringency of $\mathrm{LOD}=2$, 3 genetic loci were required to accurately assign all individuals to species. This number increased to 6 loci at $\mathrm{LOD}=3$, and all 14 loci at $\mathrm{LOD}=4$. At each level of stringency, $100 \%$ of individuals were correctly assigned to species.

The Powsim analysis of Gulf Menhaden was conducted with 15 loci and excluded the previously mentioned loci that deviated from HWE and had high null allele frequencies (BP017, BP301, AF039660, and AF039661). At an effective population size of 500, significant genetic divergence among populations was detected in all 500 simulations after 10 generations of drift $\left(F_{\mathrm{st}}=0.01\right)$, with both sample sizes. At an effective population size of $1000\left(F_{\text {st }}=0.004\right)$, significant genetic divergence was detected in $91 \%(454 / 500)$ of simulations with a sample size of 40 individuals and in 96\% (480/500) of simulations when $n=50$. At the lowest level of genetic divergence $\left(F_{\text {st }}=0.001\right)$, only $16 \%(78 / 500)$ of simulations resulted in a significant genetic divergence result when $n=40$ and $22 \%(112 / 500)$ of simulations had that result when $n=50$.

Analysis of genetic structure of Gulf Menhaden was conducted with the same 15 loci that were used in population assignment (excluding HWE deviating loci). There was little evidence for significant genetic structure among the 4 Gulf Menhaden samples. The Structure analysis indicated that $K=1$ was the most likely number of genetic clusters represented in the sample (posterior probability $P>0.999$ ). When individual admixture scores for levels of $K>1$ were examined, all individuals were approximately equal admixtures of all 4 genetic clusters. Such a finding is expected in the case of no genetic structure, and in such cases the admixture summary statistic $(\alpha)$ can vary greatly during the course of Structure runs (Pritchard et al., 2000). The AMOVA results similarly indicated a lack of genetic structure among samples $\left(F_{\mathrm{st}}=0.002, P=1.0\right)$, but there was a small but significant amount of structure among individuals within samples $\left(F_{\text {is }}=0.052, P<0.001\right)$. A post hoc examination of locus-specific $F_{\text {is }}$ indicated that 6 loci of the original suite of 15 loci had significantly 
Table 3

Statistics for 19 microsatellite loci examined in Finescale Menhaden (Brevoortia gunteri), Yellowfin Menhaden (B. smithi), and Atlantic Menhaden (B. tyrannus) collected throughout the Gulf of Mexico in 2002-04. Statistics were determined with Arlequin, vers. 3.5 (Excoffier et al., 2005). Statistics include the number of alleles, expected heterozygosity $\left(H_{\mathrm{e}}\right)$, and proportion of simulations that resulted in a more extreme test statistic for the Hardy-Weinberg equilibrium (HWE) exact test within samples (equivalent to an exact Pvalue). Statistical significance of the HWE $P$-value (initial $\alpha=0.05)$ was determined before $(*)$ and after $(* *)$ sequential Bonferroni adjustment.

\begin{tabular}{|c|c|c|c|c|c|c|c|c|c|}
\hline & \multicolumn{3}{|c|}{ Finescale Menhaden } & \multicolumn{3}{|c|}{ Yellowfin Menhaden } & \multicolumn{3}{|c|}{ Atlantic Menhaden } \\
\hline & Alleles & $H_{\mathrm{e}}$ & HWE & Alleles & $H_{\mathrm{e}}$ & HWE & Alleles & $H_{\mathrm{e}}$ & HWE \\
\hline Bp003 & 2 & 0.130 & 1.000 & 1 & 0.000 & na & 10 & 0.546 & 0.622 \\
\hline Bp017 & 2 & 0.426 & 1.000 & 2 & 0.193 & 1.000 & 8 & 0.640 & 0.073 \\
\hline Bp039 & 4 & 0.133 & 0.065 & 1 & 0.000 & na & 5 & 0.625 & 0.370 \\
\hline Bp121 & 8 & 0.719 & 0.341 & 5 & 0.700 & 0.190 & 12 & 0.825 & 0.512 \\
\hline Bp155 & 0 & 0.000 & na & 0 & 0.000 & na & 0 & 0.000 & na \\
\hline Вp221 & 5 & 0.575 & 0.318 & 3 & 0.323 & 0.489 & 9 & 0.611 & 0.363 \\
\hline Bp230 & 7 & 0.769 & 0.787 & 7 & 0.693 & 0.810 & 9 & 0.838 & $0.000 * *$ \\
\hline Bp239 & 9 & 0.862 & 0.052 & 7 & 0.660 & 0.696 & 17 & 0.927 & $0.009 *$ \\
\hline Bp275 & 6 & 0.662 & 0.091 & 3 & 0.398 & 1.000 & 14 & 0.890 & 0.056 \\
\hline Bp301 & 8 & 0.779 & 0.286 & 10 & 0.848 & $0.028^{*}$ & 11 & 0.866 & $0.010^{*}$ \\
\hline Bp473 & 2 & 0.130 & 1.000 & 3 & 0.479 & 0.720 & 4 & 0.363 & 0.118 \\
\hline Bp489 & 2 & 0.045 & 1.000 & 1 & 0.000 & na & 1 & 0.000 & na \\
\hline Bp500 & 5 & 0.355 & 0.201 & 1 & 0.000 & na & 11 & 0.813 & $0.034^{*}$ \\
\hline Bp531 & 5 & 0.593 & 0.335 & 5 & 0.599 & 0.386 & 11 & 0.889 & 0.900 \\
\hline$A F 039657$ & 2 & 0.045 & 1.000 & 2 & 0.053 & 1.000 & 12 & 0.698 & 0.231 \\
\hline AF039658 & 8 & 0.791 & 0.195 & 5 & 0.679 & 0.199 & 11 & 0.837 & $0.000 * *$ \\
\hline AF039660 & 3 & 0.519 & $0.000 * *$ & 3 & 0.280 & 1.000 & 4 & 0.550 & $0.000 * *$ \\
\hline AF039661 & 1 & 0.000 & na & 2 & 0.341 & 1.000 & 2 & 0.068 & 1.000 \\
\hline AF049462 & 9 & 0.804 & 0.620 & 7 & 0.707 & 0.148 & 19 & 0.937 & $0.004 *$ \\
\hline Overall & 4.6 & 0.439 & - & 3.6 & 0.366 & - & 8.9 & 0.628 & - \\
\hline
\end{tabular}

high values of $F_{\text {is }}$; of these, 4 loci also indicated low frequency $\left(p_{\mathrm{n}}<0.10\right)$ null alleles. The Mantel test indicated a lack of correlation between genetic divergence and geographic distance (Mantel $r=0.415, P=0.131$ ).

\section{Discussion}

The analysis of population structure in wild species can be confounded by a paucity of readily available marker loci that are designed for a specific genus or species. The genus Brevoortia is one in which previous studies have been hindered by loci that lacked adequate genetic variability (Anderson, 2007; Anderson and Karel, 2007; although, see Lynch et al., 2010). The stated purpose of this study was to develop more effective tools for genetic analysis of Brevoortia spp. that could be used in the context of stock assessments. In particular, the intended purpose of the additional loci was 1) to improve the stock definition of the menhaden fishery in the Gulf of Mexico as it pertains to the proportion of each menhaden species in the annual catch and 2) to determine whether multiple populations of
Gulf Menhaden are exploited in the fishery. Because these 2 goals require somewhat different methods, we assessed each independently in the context of the microsatellite loci described in this study.

Species identification of individuals that occur within the Gulf of Mexico menhaden fishery can be challenging. Although there are well-described morphological differences between Gulf Menhaden, Yellowfin Menhaden, and Finescale Menhaden, differences in morphological characteristics are subtle and can potentially lead to inaccurate identification (Dahlberg, 1970; Anderson and McDonald, 2007). Genetic differentiation has been equally problematic. Anderson (2007) described reciprocal monophyletic mitochondrial DNA lineages between Gulf Menhaden and both of its congeners in the Gulf of Mexico. However, this same mtDNA analysis indicated that Finescale Menhaden and Yellowfin Menhaden could not be reliably distinguished with mtDNA.

Species identification through the use of mtDNA loci can be troublesome when closely related species are hybridizing, as is the case with Gulf Menhaden and Yellowfin Menhaden (Turner, 1969; Dahlberg, 1970; An- 


\section{Table 4}

Statistics from the Whichloci analysis of 14 microsatellite loci in the genus Brevoortia. The average rank, locus score, relative score (the power of an individual locus for species discrimination, relative to the power of all 14 loci), and cumulative score were calculated from locus scores across 100 replicated simulations of species assignment for Gulf Menhaden (Brevoortia patronus), Finescale Menhaden (B. gunteri), or Yellowfin Menhaden (B. smithi). The LOD (log odds) columns indicate whether inclusion of a locus was necessary ("1") or not necessary (“0") for correct assignment at the given level of stringency.

\begin{tabular}{lccccccc}
\hline Locus & Avg. rank & Score & Relative score (\%) & Cumulative score & LOD=2 & LOD=3 & LOD=4 \\
\hline AF039657 & 1 & 0.938 & 18.45 & 18.45 & 1 & 1 & 1 \\
Bp500 & 2 & 0.900 & 17.70 & 36.15 & 1 & 1 & 1 \\
Bp473 & 3 & 0.763 & 15.00 & 51.14 & 1 & 1 & 1 \\
Bp531 & 4 & 0.701 & 13.79 & 64.93 & 0 & 1 & 1 \\
AF049462 & 5 & 0.384 & 7.54 & 72.48 & 0 & 1 & 1 \\
Bp489 & 6 & 0.324 & 6.38 & 78.85 & 0 & 1 & 1 \\
Bp003 & 7 & 0.252 & 4.95 & 83.80 & 0 & 0 & 1 \\
Bp039 & 8 & 0.238 & 4.67 & 8.48 & 0 & 0 & 1 \\
Bp121 & 9 & 0.209 & 4.11 & 92.59 & 0 & 0 & 1 \\
Bp221 & 10 & 0.183 & 3.60 & 96.18 & 0 & 0 & 1 \\
Bp239 & 11 & 0.117 & 2.30 & 98.48 & 0 & 0 & 1 \\
Bp275 & 12 & 0.071 & 1.39 & 99.88 & 0 & 0 & 1 \\
AF039658 & 13 & 0.006 & 0.12 & 100.00 & 0 & 0 & 1 \\
Bp230 & 14 & 0.000 & 0.00 & 100.00 & 0 & 0 & 1 \\
\end{tabular}

derson and Karel, 2007) and potentially could be the case with Gulf Menhaden and Finescale Menhaden (Anderson and McDonald, 2007). In the case of hybrids of Gulf Menhaden and Yellowfin Menhaden, F1 individuals usually carry the mtDNA haplotype of Yellowfin Menhaden. Assuming that hybridization is common in the eastern Gulf of Mexico, mtDNA species identification potentially would lead to overestimation of the presence of Yellowfin Menhaden in catch data if hybrid populations are encountered because hybrids would be identified incorrectly as pure Yellowfin Menhaden. Hybrids were not encountered in our current study; however, to obtain individuals of unequivocal taxonomic classification, sampling was directed toward locales where hybridization had not previously been observed.

The uncertainty in mtDNA monophyly among Brevoortia species led Lynch et al. (2010) to question the taxonomic validity of Gulf Menhaden and Atlantic Menhaden. Given this uncertainty, species identification in these species by DNA analysis would be accomplished most reliably with multiple co-dominant loci. The analysis of Anderson and Karel (2007) indicated that species identification in the presence of hybrids could be achieved with as few as 5 microsatellites, although there was some uncertainty in estimation of levels of introgression in hybrid individuals.

In our study, the 14 loci used in simulated speciesidentification analysis (Powsim analysis) included not only 3 of the loci used in Anderson and Karel (2007) but also an additional 11 loci, some of which performed at least as well in species identification as the original loci. The Whichloci analysis indicated that the 6 most informative loci in this group could be used to distinguish between Gulf Menhaden, Yellowfin Menhaden, and Finescale Menhaden with 100\% accuracy, and with a statistical error rate of $\alpha=0.001$. Accuracy in species identification can be achieved with a statistical error rate of $\alpha=0.0001$ given use of all 14 loci. These loci, therefore, represent a powerful tool in species identification, and can be used to reliably distinguish all Gulf of Mexico species (and hybrids) for the purpose of future stock enhancements.

To our knowledge, only a single previous study has attempted to explicitly examine the genetic structure of Gulf Menhaden in the Gulf of Mexico with intensive sampling. ${ }^{3}$ This earlier study was conducted with $5 \mathrm{mi}-$ crosatellite loci and a single mtDNA locus; the Powsim analysis used here indicates that this level of genetic sampling is inadequate to answer questions about finescale population structure in this species. Success in assignment of individuals to populations is a function of both the number of loci used and the polymorphism (number of alleles) at each locus (Bernatchez and Duchesne, 2000). The loci described here improve both of those aspects of population assignment and represent a significant improvement in the tools available

\footnotetext{
${ }^{3}$ Anderson, J. D. 2006. Conservation genetics of Gulf Menhaden (Brevoortia patronus): implications for the management of a critical forage component for Texas coastal gamefish ecology. Federal Aid in Sportfish Restoration Act Technical Series, F-144-R, 34 pp.
} 
to managers in assessment of population structure in the Gulf Menhaden. An analysis of genetic structure in Gulf Menhaden using these loci showed no evidence for discreet populations and no evidence for isolation-bydistance across a majority of the range of the species in the northern Gulf of Mexico. It has long been suspected that genetic divergence among Gulf Menhaden samples throughout the Gulf of Mexico is very low, and, therefore, stock assessments have generally assumed that the menhaden fishery in the Gulf of Mexico is centered upon a single fishery stock. Although the results of this study are a compelling validation of that assumption, the small sample sizes examined here relegate this finding as preliminary rather than conclusive. The power analysis indicated that sample sizes of 50 or more are necessary to conclusively detect very low levels of genetic divergence $\left(F_{\text {st }} \leq 0.004\right)$ through the use of all 15 markers for Gulf Menhaden.

Although the loci described in this article are polymorphic in other species of Brevoortia, the power of these markers for fine-scale population assessments in these species was not rigorously tested. The polymorphism exhibited by samples of Gulf Menhaden at these loci was not repeated to the same magnitude in other congeners. The finding that other Brevoortia species exhibit relatively low genetic variation at loci originally discovered in Gulf Menhaden is not surprising. Ascertainment bias, or decreased genetic variability in species that were not directly targeted in the original marker selection process, is a common finding in genetic studies of microsatellite loci among closely related species (Ellegren et al., 1997: Hutter et al., 1998; Vowles and Amos, 2005). Both heterozygosity and PIC scores were lower in all 3 Brevoortia congeners.

The ascertainment biases in comparisons that involve Gulf Menhaden and either Finescale Menhaden or Yellowfin Menhaden were more profound than were the biases in comparisons between Gulf Menhaden and Atlantic Menhaden. In the former 2 comparisons, there were qualitative differences in the median PCR fragment lengths in direct comparisons of species (Gulf Menhaden generally had longer alleles) and significantly higher genetic variability in the focal species (Gulf Menhaden exhibited higher PIC and $H_{\mathrm{e}}$ ). In contrast, PCR fragment lengths were generally similar between Gulf Menhaden and Atlantic Menhaden, although there was a significant difference in $H_{\mathrm{e}}$ and PIC. Similar median repeat lengths between Gulf Menhaden and Atlantic Menhaden likely reflect a shallow evolutionary lineage between these species (Bowen and Avise, 1990; Anderson, 2007), in contrast to relatively deep taxonomic divergence between Gulf Menhaden and both Yellowfin Menhaden and Finescale Menhaden (Anderson, 2007). This result not only underscores the notion that the loci described here will be useful in future studies of both Gulf Menhaden and Atlantic Menhaden but also indicates that the use of loci in studies of Yellowfin Menhaden and Finescale Menhaden should be undertaken with caution.

\section{Conclusions}

It has been suggested previously that the main species exploited in the menhaden fishery of the Gulf of Mexico is the Gulf Menhaden (Ahrenholz, 1981). Because of the importance of this fishery in the Gulf of Mexico and because of the significance of Gulf Menhaden to inshore trophic systems, this finding should be re-assessed. In particular, the proportional contribution of each menhaden species occurring in the Gulf of Mexico to the annual catch should be examined through the use of a systematic method. Moreover, the presence of a single population of Gulf Menhaden throughout the Gulf of Mexico has been assumed in recent stock assessments, although this assumption has not been rigorously tested. The species composition and the number of exploited Gulf Menhaden populations can be reliably assessed with genetic data. The gene loci described in this study can be used to address both of these concerns, and they represent an improvement in the tools available for examination of the population structure of the genus Brevoortia.

\section{Acknowledgments}

This work was funded by the Texas Parks and Wildlife Department, Coastal Fisheries Division (TPWD-CF). We wish to thank D. McDonald for helpful comments on the first draft. An internal TPWD-CF review was conducted by M. Fisher and 3 anonymous reviewers. Reference to trade names does not imply endorsement by the state of Texas.

\section{Literature cited}

Ahrenholz, D. W.

1981. Recruitment and exploitation of gulf menhaden, Brevoortia patronus. Fish. Bull. 79:325-335.

Anderson, J. D.

2007. Systematics of the North American menhadens: molecular evolutionary reconstructions in the genus Brevoortia (Clupeiformes: Clupeidae). Fish. Bull. 105:368-378.

Anderson, J. D., and W. J. Karel.

2007. Genetic evidence for asymmetric hybridization between menhadens (Brevoortia spp.) from peninsular Florida. J. Fish Biol. 71:235-249.

Anderson, J. D., and D. L. McDonald.

2007. Morphological and genetic investigations of two western Gulf of Mexico menhadens (Brevoortia spp.). J. Fish Biol. 70(S):139-147.

Banks, M. A., W. Eichert, and J. B. Olsen.

2003. Which genetic loci have greater population assignment power? Bioinformatics 19:1436-1438.

Begg, G. A., and J. R. Waldman.

1999. An holistic approach to fish stock identification. Fish. Res. 43:35-44. 
Bernatchez, L., and P. Duchesne.

2000. Individual-based genotype analysis in studies of parentage and population assignment: how many loci, how many alleles? Can. J. Fish Aquat. Sci. 57:1-12.

Botstein D., R. L. White, M. Skolnick, and R. W. Davis. 1980. Construction of a genetic linkage map in man using restriction fragment length polymorphisms. Am. J. Hum. Genet. 32:314-331.

Bowen, B. W., and J. C. Avise.

1990. Genetic structure of Atlantic and Gulf of Mexico populations of sea bass, menhaden and sturgeon: influence of zoogeographic factors and life-history patterns. Mar. Biol. 107:371-381.

Brookfield, J. F. Y.

1996. A simple new method for estimating null allele frequency from heterozygote deficiency. Mol. Ecol. 5:453-455.

Carvalho, G. R., and L. Hauser.

1994. Molecular genetics and the stock concept in fisheries. Rev. Fish Biol. Fish. 4:326-350.

Chakraborty, R., M. De Andrade, S. P. Daiger, and B. Budowle. 1992. Apparent heterozygote deficiencies observed in DNA typing data and their implications in forensic applications. Ann. Hum. Genet. 56:45-47.

Dahlberg, M. D.

1970. Atlantic and Gulf of Mexico menhadens, genus Brevoortia (Pisces: Clupeidae). Bull. Fla. State Mus., Biol. Sci. 15:91-162.

Ellegren, H., S. Moore, N. Robinson, K. Byrne, W. Ward, and

B. C. Sheldon.

1997. Microsatellite evolution-a reciprocal study of repeat lengths at homologous loci in cattle and sheep. Mol. Biol. Evol. 14:854-860.

Excoffier, L., P. Smouse, and J. Quattro.

1992. Analysis of molecular variance inferred from metric distances among DNA haplotypes: application to human mitochondrial DNA restriction data. Genetics 131:479-491.

Excoffier, L., and M. Slatkin,

1998. Incorporating genotypes of relatives into a test of linkage disequilibrium. Am. J. Hum. Genet. 62:171-180.

Excoffier, L., G. Laval, and S. Schneider.

2005. Arlequin ver. 3.0: An integrated software package for population genetics data analysis. Evol. Bioinform. Online 1:47-50.

Glenn, T. C., and N. A. Schable.

2005. Isolating microsatellite DNA loci. Methods Enzymol. 395:202-222.

Goudet, J.

1995. FSTAT (vers. 1.2): a computer program to calculate F-statistics. J. Hered. 86:485-486.

Guo, S., and E. Thompson.

1992. Performing the exact test of Hardy-Weinberg proportion for multiple alleles. Biometrics 48:361-372.

Hutter, C. M., M. D. Schug, and C. F. Aquadro.

1998. Microsatellite variation in Drosophila melanogaster and Drosophila simulans: a reciprocal test of the ascertainment bias hypothesis. Mol. Biol. Evol. $15: 1620-1636$.

Kalinowski, S. T.

2004. Genetic polymorphism and mixed-stock fisheries analysis. Can. J. Fish. Aquat. Sci. 61:1075-1082.

Lynch, A. J., J. R. McDowell, and J. E. Graves.

2010. A molecular genetic investigation of the population structure of Atlantic menhaden (Brevoortia tyrannus). Fish. Bull. 108:87-97.

Pritchard, J. K., M. Stephens, and P. Donnelly.

2000. Inference of population structure using multilocus genotype data. Genetics 155:945-959.

Portnoy, D. S., and J. R. Gold.

2012. Evidence of multiple vicariance in a marine suturezone in the Gulf of Mexico. J. Biogeogr. 39:1499-1507.

R Development Core Team.

2011. R: a language and environment for statistical computing. R Foundation for Statistical Computing, Vienna, Austria. [Available from http://www.R-project.org.]

Rice, W. R.

1989. Analyzing tables of statistical tests. Evolution 43:223-225.

Rico, C., I. Rico, and G. Hewitt.

1996. 470 million years of conservation of microsatellite loci among fish species. Proc. R. Soc. Lond., Ser. B: Biol. Sci. 263:549-557.

Ryman, N., and S. Palm.

2006. POWSIM: a computer program for assessing statistical power when testing for genetic differentiation. Mol. Ecol. Notes 6:600-602.

Slatkin, M.

1995. A measure of population subdivision based on microsatellite allele frequencies. Genetics 139:457-462.

Turner, W. R.

1969. Life history of menhadens in the eastern Gulf of Mexico. Trans. Am. Fish. Soc. 98:216-224.

Van Oosterhout, C., W. F. Hutchinson, D. P. M. Willis, and P. Shipley.

2004. MICRO-CHECKER: software for identifying and correcting genotyping errors in microsatellite data. Mol. Ecol. Notes 4:535-538.

Van Oosterhout, C., D. Weetman, and W. F. Hutchinson.

2006. Estimation and adjustment of microsatellite null alleles in nonequilibrium populations. Mol. Ecol. Notes $5: 255-256$

Vowles, E. J., and W. Amos.

2005. Quantifying ascertainment bias and species-specific length differences in human and chimpanzee microsatellites using genome sequencs. Mol. Biol. Evol. 23:598-607.

Waples, R. S., A. E. Punt, and J. M. Cope.

2008. Integrating genetic data into management of marine resources: how can we do it better? Fish Fish. 9:423-449

Waters, J. M., J. M. Epifanio, T. Gunter, and B. L. Brown. 2000. Homing behaviour facilitates subtle genetic differentiation among river populations of Alosa sapidissima: microsatellites and mtDNA. J. Fish Biol. 56:622-636. 